\title{
On the calculation of reflectance in non-uniform ear canals
}

\author{
Nørgaard, Kren Rahbek; Charaziak, Karolina K. ; Shera, Christopher A.
}

Published in:

Journal of the Acoustical Society of America

Link to article, DOI:

$10.1121 / 1.5124000$

Publication date:

2019

Document Version

Publisher's PDF, also known as Version of record

Link back to DTU Orbit

Citation (APA):

Nørgaard, K. R., Charaziak, K. K., \& Shera, C. A. (2019). On the calculation of reflectance in non-uniform ear canals. Journal of the Acoustical Society of America, 146(2), 1464-1474. https://doi.org/10.1121/1.5124000

\section{General rights}

Copyright and moral rights for the publications made accessible in the public portal are retained by the authors and/or other copyright owners and it is a condition of accessing publications that users recognise and abide by the legal requirements associated with these rights.

- Users may download and print one copy of any publication from the public portal for the purpose of private study or research.

- You may not further distribute the material or use it for any profit-making activity or commercial gain

- You may freely distribute the URL identifying the publication in the public portal

If you believe that this document breaches copyright please contact us providing details, and we will remove access to the work immediately and investigate your claim. 
See discussions, stats, and author profiles for this publication at: https://www.researchgate.net/publication/335460131

\section{On the calculation of reflectance in non-uniform ear canals}

Article in The Journal of the Acoustical Society of America · August 2019

Dol: $10.1121 / 1.5124000$

3 authors, including:

Kren Monrad Nørgaard

15. Interacoustics $\mathrm{A} / \mathrm{S}$

9 PUBLICATIONS 41 CITATIONS

SEE PROFILE

Karolina Kinga Charaziak

(7) University of Southern California

37 PUBLICATIONS 179 CITATIONS

SEE PROFILE 


\title{
On the calculation of reflectance in non-uniform ear canals
}

\author{
Kren Rahbek Nørgaard ${ }^{\text {a) }}$ \\ Acoustic Technology, Department of Electrical Engineering, Technical University of Denmark, Ørsteds Plads, \\ Building 352, Kongens Lyngby, DK-2800, Denmark \\ Karolina K. Charaziak and Christopher A. Shera ${ }^{\text {b) }}$ \\ Caruso Department of Otolaryngology, University of Southern California, 1640 Marengo Street, Los Angeles, \\ California 90033, USA
}

(Received 12 March 2019; revised 2 August 2019; accepted 8 August 2019; published online 28 August 2019)

\begin{abstract}
Ear-canal reflectance is useful for quantifying the conductive status of the middle ear because it can be measured non-invasively at a distance from the tympanic membrane. Deriving the ear-canal reflectance requires decomposing the total acoustic pressure into its forward- and reversepropagating components. This decomposition is conveniently achieved using formulas that involve the input and characteristic impedances of the ear canal. The characteristic impedance is defined as the ratio of sound pressure to volume flow of a propagating wave and, for uniform waveguides, the plane-wave characteristic impedance is a real-valued constant. However, in non-uniform waveguides, the characteristic impedances are complex-valued quantities, depend on the direction of propagation, and more accurately characterize a propagating wave in a non-uniform ear canal. In this paper, relevant properties of the plane-wave and spherical-wave characteristic impedances are reviewed. In addition, the utility of the plane-wave and spherical-wave reflectances in representing the reflection occurring due to the middle ear, calibrating stimulus levels, and characterizing the emitted pressure in simulated non-uniform ear canals is investigated and compared.
\end{abstract}

(C) 2019 Acoustical Society of America. https://doi.org/10.1121/1.5124000

[ICB]

Pages: $1464-1474$

\section{INTRODUCTION}

Although the acoustic impedance of the tympanic membrane characterizes the conductive status of the middle ear, it is difficult to measure in human ears. A more practical alternative measure for representing essentially the same physical information is the ear-canal reflectance, which has the virtue that its magnitude is independent of the distance between the measurement location and the tympanic membrane in a uniform ear canal. The ear-canal reflectance has proved useful for identifying conductive hearing disorders (Ellison et al., 2012; Feeney and Keefe, 2001; Keefe et al., 2000; Keefe et al., 2012; Merchant et al., 2014; Piskorski et al., 1999), calibrating stimulus levels (Lewis et al., 2009; McCreery et al., 2009; Scheperle et al., 2011, 2008; Souza et al., 2014; Withnell et al., 2009), and estimating the emitted pressure from the ear in the form of otoacoustic emissions (OAEs) (Charaziak and Shera, 2017). The pressure reflectance is defined as the ratio of reverse- to forwardpropagating pressure waves and its measurement therefore requires decomposing the total acoustic pressure into these components. In practice, the reflectance is easily calculated from the input impedance and the characteristic impedances.

The characteristic impedance is defined as the ratio of sound pressure to volume flow of a propagating wave. In a lossless uniform waveguide, the plane-wave characteristic

\footnotetext{
a) Also at: Interacoustics A/S, Audiometer Allé 1, Middelfart, DK-5500, Denmark. Electronic mail: krng@interacoustics.com

b) Also at: Department of Physics and Astronomy, University of Southern California, Los Angeles, CA 90089, USA.
}

impedance is a frequency-independent real-valued quantity and has convenient time-domain properties that allow it to be estimated in situ from the measured input impedance (Keefe et al., 1992; Nørgaard et al., 2017; Rasetshwane and Neely, 2011). By contrast, the characteristic impedances of non-uniform waveguides are complex-valued, depend both on frequency and on the direction of propagation, and have time-domain properties that differ considerably from the plane-wave characteristic impedance (e.g., Agulló et al., 1988). The characteristic impedances of certain acoustic horns, governed by the Webster horn equation (Webster, 1919), with independently propagating waves can be found analytically. However, characteristic impedances can also be formulated for arbitrary geometries using the WKB (Wentzel-KramersBrillouin) approximation (Keefe and Barjau, 1999), although propagating waves cannot be assumed to exist at all locations or frequencies.

In ear canals, the plane-wave reflectance is usually calculated from the input impedance-measured using an ear probe (e.g., Keefe et al., 1992; Voss and Allen, 1994)—and estimates of the ear canal's plane-wave characteristic impedance. Farmer-Fedor and Rabbitt (2002) noted that, because ear canals are inherently non-uniform, the assumption of a planewave sound field may not always be valid. Consequently, the forward- and reverse-propagating pressure- and volume-flow components are more accurately related by such non-planar characteristic impedances. However, Farmer-Fedor and Rabbitt (2002) did not clarify how the ear-canal reflectance should be calculated from the measured ear-canal impedance in a practical setup if not using the plane-wave characteristic impedance. 
In this paper, we assess the application of spherical-wave characteristic impedances for calculating the ear-canal reflectance and investigate whether they provide an improvement in quantifying reflections from the middle ear. For this, we compute reflectances in simulated non-uniform ear canals based on representative measurements of ear-canal geometry reported by Egolf et al. (1993). The analyses are carried out based on simulations that, as far as possible, represent what could be measured in a real ear canal using existing measurement methods. In addition, we investigate whether the spherical-wave ear-canal reflectance provides a basis for improving the in-situ calibration of stimulus levels and quantifying the emitted pressure during OAE measurements.

\section{BACKGROUND}

\section{A. Pressure reflectance}

Assuming the existence of one-dimensional propagating waves, the pressure reflection coefficient, or reflectance, $\Gamma(\omega, x),{ }^{1}$ as a function of the angular frequency $\omega$ at any point $x$ along a one-dimensional transmission line is defined as the ratio of reverse-propagating $p_{-}(\omega, x)$ to forwardpropagating $p_{+}(\omega, x)$ pressure components,

$$
\Gamma(\omega, x)=\frac{p_{-}(\omega, x)}{p_{+}(\omega, x)} .
$$

The total acoustic pressure is $p(\omega, x)=p_{+}(\omega, x)+p_{-}(\omega, x)$. The forward and reverse characteristic impedances $Z_{ \pm}(\omega, x)$ are defined as the ratios of forward- and reverse-propagating pressure $p_{ \pm}(\omega, x)$ to volume flow $u_{ \pm}(\omega, x)$ components,

$$
Z_{ \pm}(\omega, x)= \pm \frac{p_{ \pm}(\omega, x)}{u_{ \pm}(\omega, x)}
$$

The input impedance $Z_{\text {in }}(\omega, x)$ of the transmission line is

$$
Z_{\text {in }}(\omega, x)=\frac{p(\omega, x)}{u(\omega, x)}=\frac{p_{+}(\omega, x)+p_{-}(\omega, x)}{u_{+}(\omega, x)+u_{-}(\omega, x)}
$$

The total acoustic volume flow is $u(\omega, x)=u_{+}(\omega, x)$ $+u_{-}(\omega, x){ }^{2}$ By combining Eqs. (1)-(3), the reflectance $\Gamma(\omega, x)$ can be expressed in terms of the input impedance $Z_{\text {in }}(\omega, x)$ and the characteristic impedances $Z_{ \pm}(\omega, x)$,

$$
\Gamma(\omega, x)=\frac{Z_{\text {in }}(\omega, x) / Z_{+}(\omega, x)-1}{Z_{\text {in }}(\omega, x) / Z_{-}(\omega, x)+1} .
$$

\section{B. The Webster horn equation and characteristic impedances}

The characteristic impedances $Z_{ \pm}(\omega, x)$ of certain types of horns can be obtained analytically using the Webster horn equation (Webster, 1919), which governs one-dimensional pressure waves $p(\omega, x)$ of varying equipotential area $A(x)$ along the longitudinal coordinate $x$,

$$
p^{\prime \prime}(\omega, x)+[\log A(x)]^{\prime} p^{\prime}(\omega, x)+k^{2}(\omega) p(\omega, x)=0 .
$$

The primes denote differentiation with respect to $x$ and the free-field wavenumber $k(\omega)=\omega / c$, where $c$ is the speed of sound. Salmon (1946a) showed that exact solutions to Eq. (5) exist consisting of freely propagating waves for certain area functions $A(x)$, namely conical, catenoidal, exponential, and hyperbolic. ${ }^{3}$ By assuming planar wavefronts [i.e., by taking $A(x)$ to be the planar cross-sectional area of the physical conduit], Salmon (1946b) showed that Eq. (5) can be used to predict the acoustic behavior of non-uniform waveguides. Salmon (1946b) also formulated the reduced horn equation by including the inverse magnitude dependency of a spherical wave from its source in the variable $\psi(\omega, x)$ $=p(\omega, x) \sqrt{A(x)}$,

$$
\psi^{\prime \prime}(\omega, x)+\kappa^{2}(\omega, x) \psi(\omega, x)=0 .
$$

Here, $\kappa^{2}(\omega, x)=k^{2}(\omega)-U(x)$ and

$$
U(x)=\frac{r^{\prime \prime}(x)}{r(x)},
$$

is called the horn function with the effective radius $r(x)$ such that $A(x)=r^{2}(x) \pi$. Benade and Jansson (1974) showed that the horn function $U(x)$ depends strongly on whether the waves inside the horn are assumed to be planar versus spherical, resulting in substantially different solutions to the acoustic pressure. Agulló et al. (1999) extended the analysis by introducing the curvilinear horn equation using a curvilinear coordinate system on axisymmetrical horns. Generally, if the area function $A(x)$ is understood to characterize the equipotential surfaces of the wavefronts rather than the geometrical cross-sectional area, Eq. (5) is not limited to wavefronts of any particular geometry (Putland, 1993). Among the different horn profiles that satisfy Eq. (5), we consider planar and conical cases in this paper.

The plane-wave characteristic impedance $Z_{0}$ characterizes a propagating plane wave and, at any given point along a lossless uniform transmission line, it can be represented in terms of the air density $\rho$, speed of sound $c$, and constant planar cross-sectional area $A^{\mathrm{pl}}$,

$$
Z_{ \pm}^{\mathrm{pl}}(\omega, x) \equiv Z_{0}=\frac{\rho c}{A^{\mathrm{pl}}}
$$

independent of location and direction, where the pl superscript denotes plane waves. The real-valued frequency-independent nature of the plane-wave characteristic impedance $Z_{0}$ results in conveniently localized features at $t=0$ in the time-domain plane-wave characteristic impedance $z_{0}(t)$,

$$
z_{ \pm}^{\mathrm{pl}}(t, x) \equiv z_{0}(t)=\mathcal{F}^{-1}\left[Z_{0}\right]=Z_{0} \delta(t) .
$$

Here, $\mathcal{F}^{-1}[\cdot]$ is the inverse Fourier transform, $\delta(\cdot)$ is the Dirac delta function, and the dot denotes an arbitrary argument.

For spherical waves propagating in a conical waveguide, the spherical-wave characteristic impedances are complexvalued frequency-dependent quantities that depend on location and direction of propagation. The forward and reverse spherical-wave characteristic impedances $Z_{ \pm}^{\mathrm{sph}}(\omega, x)$ were reported by Benade (1988), 


$$
Z_{ \pm}^{\mathrm{sph}}(\omega, x)=Z_{0}(x) \| \pm j \omega L(x),
$$

where the sph superscript denotes spherical waves, $\|$ denotes the parallel coupling, the inertance $L(x)=2 \rho /\left[A^{\text {sph }}(x)\right]^{\prime}{ }^{4}$ and the local plane-wave characteristic impedance

$$
Z_{0}(x)=\frac{\rho c}{A^{\operatorname{sph}}(x)},
$$

corresponding to the spherical-cap area $A^{\mathrm{sph}}(x)$.

The representation of the spherical-wave characteristic impedances in the time domain is associated with a number of problems that make them unfit for some practical applications. Previous authors (Agulló et al., 1988; Martínez and Agulló, 1988; Martínez et al., 1988) studied reflections between conical waveguides of different taper and noted that the time-domain spherical-wave characteristic impedances $z_{ \pm}^{\mathrm{sph}}(t, x)$ take the form

$$
z_{ \pm}^{\mathrm{sph}}(t, x)=\mathcal{L}^{-1}\left[Z_{ \pm}^{\mathrm{sph}}(s, x)\right]=Z_{0}(x)\left(\delta(t) \mp \frac{e^{\mp t / \tau(x)}}{\tau(x)} \theta(t)\right),
$$

with the inverse unilateral Laplace transform $\mathcal{L}^{-1}[\cdot]$, the complex frequency $s=\alpha+j \omega$, the time constant $\tau(x)=L(x) /$ $Z_{0}(x)$, and the Heaviside step function $\theta(\cdot)$. Agulló et al. (1990) and Gilbert et al. (1990) pointed out the growingexponential form of the time-domain apical spherical-wave characteristic impedance $z_{ \pm}^{\mathrm{sph}}(t, x)$ for $L(x) \lessgtr 0$ (i.e., looking toward the apex of the horn). This problematic behavior occurs due to the physical impossibility of an anechoic contracting conical waveguide; in any physical horn, the growing exponential is quickly quenched by reflections at the apex. They also noted that evaluating the time-domain apical spherical-wave characteristic impedance using the inverse Fourier transform $\mathcal{F}^{-1}[\cdot]$ results is a non-causal function due to the time-reversal property of the complex conjugate, $Z_{ \pm}^{\mathrm{sph}}(\omega, x)=\left[Z_{\mp}^{\mathrm{sph}}(\omega, x)\right]^{*}$, and the Fourier transform,

$$
\begin{aligned}
z_{ \pm}^{\mathrm{sph}}(t, x) & =\mathcal{F}^{-1}\left[Z_{ \pm}^{\mathrm{sph}}(\omega, x)\right] \\
& =Z_{0}(x)\left(\delta(t)-\frac{e^{\mp t / \tau(x)}}{\tau(x)} \theta( \pm t)\right),
\end{aligned}
$$

because the Fourier transform of a growing exponential does not exist. Gilbert et al. (1990) argued that the difference between time-domain spherical-wave characteristic impedances $z_{ \pm}^{\text {sph }}(t, x)$ evident between Eqs. (12) and (13) occurs due to the different assumptions imposed onto the time-domain signal by the inverse unilateral Laplace and Fourier transforms. The unilateral Laplace transform assumes a causal function and can handle systems that are not bounded-input boundedoutput stable in the time domain. Conversely, the Fourier transform is undefined for unstable signals but imposes no assumption of causality. ${ }^{5}$ The non-causality arising due to the inverse Fourier transform in Eq. (13) has implications on time-domain analysis of the ear-canal reflectance because the inverse Laplace transform cannot be evaluated numerically.
Keefe and Barjau (1999) derived the WKB approximation for the reduced horn equation [Eq. (6)] and showed that the corresponding WKB characteristic impedances are identical to those of spherical waves [Eq. (10)]. However, near turning points (Farmer-Fedor and Rabbitt, 2002), which occur at critical frequencies

$$
\omega_{\mathrm{c}}(x)= \begin{cases}c \sqrt{U(x)} & \text { for } U(x) \geq 0 \\ 0 & \text { for } U(x)<0\end{cases}
$$

the pressure waves transition from propagating to evanescent at a given location and the accuracy of the WKB approximation may be limited.

\section{Ear-canal reflectance}

In ear canals, the sound field is often assumed to consist of plane waves. When this approximation is valid, the characteristic impedance is independent of direction of propagation and the plane-wave reflectance $\Gamma^{\mathrm{pl}}(\omega, x)$, calculated from the input impedance $Z_{\text {in }}(\omega, x)$, takes the well-known form

$$
\Gamma^{\mathrm{pl}}(\omega, x)=\frac{Z_{\text {in }}(\omega, x)-Z_{0}(x)}{Z_{\text {in }}(\omega, x)+Z_{0}(x)},
$$

Farmer-Fedor and Rabbitt (2002) noted that, because ear canals are inherently non-uniform, the plane-wave reflectance $\Gamma^{\mathrm{pl}}(\omega, x)$ does not represent the reflectance of any realistic ear canal. Assuming a local spherical-wave sound field (or the WKB approximation), where the characteristic impedances depend on direction, the spherical-wave reflectance is

$$
\Gamma^{\mathrm{sph}}(\omega, x)=\frac{Z_{\text {in }}(\omega, x) / Z_{+}^{\mathrm{sph}}(\omega, x)-1}{Z_{\mathrm{in}}(\omega, x) / Z_{-}^{\mathrm{sph}}(\omega, x)+1} .
$$

More generally, decomposing the total pressure into its forward- and reverse-propagating components in a conical horn requires the forward and reverse spherical-wave characteristic impedances. Because ear canals can have complicated non-uniform geometries, it is reasonable to expect that the spherical-wave characteristic impedances provide a more accurate decomposition of the total pressure. ${ }^{6}$

As previously mentioned, analytical characteristic impedances can be found for any area function that provides a solution to the Webster horn equation consisting of independent wave components, including Salmon's family of horns (Salmon, 1946a). With the exceptions of the uniform and conical horns, the characteristic impedances corresponding to these remaining horns have a cut-off frequency below which the characteristic impedances become imaginary, the independent waves evanescent, and the reflectance magnitude no longer constant throughout the horn. For these reasons, we have not considered these additional characteristic impedances of Salmon's family of horns in the present analysis. A general expression for the characteristic impedances for Salmon's family of horns was reported by Leach (1996). 
Note that the cut-off frequency is equivalent to the critical frequency $\omega_{\mathrm{c}}(x)$ [Eq. (14)] when the horn function $U(x)[\mathrm{Eq}$. (7)] is a positive constant so that the transition from propagating to evanescent waves occurs at the same frequency throughout the horn.

\section{METHODS}

\section{A. Ear-canal modeling}

We modeled analytical one-dimensional ear canals of axial length $l^{\mathrm{pl}}=1.6 \mathrm{~cm}$ using $N=160$ concatenated conical horn segments, terminated by a tympanic-membrane impedance $Z_{\mathrm{tm}}(\omega)$ as illustrated in Fig. 1. We chose this subdivision arbitrarily to obtain a constant axial segment spacing of $h^{\mathrm{pl}}=0.1 \mathrm{~mm}$ such that the wavelengths at all considered frequencies up to $20 \mathrm{kHz}$ were much larger than the spacing $\lambda \gg h^{\mathrm{pl}}$. Note that for variables with spatial dependency, we replaced the longitudinal variable $x$ with the segment-number subscript $n$ for our methods and results; for practical reasons, the first segment was $n=0$. Using the two-port transfer-matrix elements $a_{i j, n}(\omega)$ of the ear canal as seen from the $n$th segment, the input impedance

$$
Z_{\mathrm{in}, n}(\omega)=\frac{a_{11, n}(\omega) Z_{\mathrm{tm}}(\omega)+a_{12, n}(\omega)}{a_{21, n}(\omega) Z_{\mathrm{tm}}(\omega)+a_{22, n}(\omega)} .
$$

From the transfer-matrix elements of each individual $m$ th segment $a_{i j, m}(\omega)$,

$$
\left[\begin{array}{ll}
a_{11, n}(\omega) & a_{12, n}(\omega) \\
a_{21, n}(\omega) & a_{22, n}(\omega)
\end{array}\right]=\prod_{m=n}^{N-1}\left[\begin{array}{ll}
a_{11, m}(\omega) & a_{12, m}(\omega) \\
a_{21, m}(\omega) & a_{22, m}(\omega)
\end{array}\right] .
$$

Analytical expressions for the transfer-matrix elements of a conical horn segment were reported by Caussé et al. (1984) and Benade (1988). They are given here in terms of the planar radii $r_{m}^{\mathrm{pl}}$ (rather than apical distances) such that the planar radius at the end of each horn segment is identical to the planar radius at the entrance of the next segment,

$$
\begin{aligned}
& a_{11, m}(\omega)=\frac{r_{m+1}^{\mathrm{pl}}}{r_{m}^{\mathrm{pl}}} \cos k(\omega) h_{m}^{\mathrm{sph}}-\frac{r_{m+1}^{\mathrm{pl}}-r_{m}^{\mathrm{pl}}}{k(\omega) r_{m}^{\mathrm{pl}} h_{m}^{\mathrm{sph}}} \sin k(\omega) h_{m}^{\mathrm{sph}}, \\
& a_{12, m}(\omega)=\frac{r_{m}^{\mathrm{pl}}}{r_{m+1}^{\mathrm{pl}}} j Z_{0, m} \sin k(\omega) h_{m}^{\mathrm{sph}}, \\
& a_{21, m}(\omega)=\frac{j}{Z_{0, m}}\left\{\left[\frac{r_{m+1}^{\mathrm{pl}}}{r_{m}^{\mathrm{pl}}}+\left(\frac{r_{m+1}^{\mathrm{pl}}-r_{m}^{\mathrm{pl}}}{k(\omega) r_{m}^{\mathrm{pl}} h_{m}^{\mathrm{sph}}}\right)^{2}\right] \sin k(\omega) h_{m}^{\mathrm{sph}}-\frac{h_{m}^{\mathrm{sph}}}{k(\omega)}\left(\frac{r_{m+1}^{\mathrm{pl}}-r_{m}^{\mathrm{pl}}}{r_{m+1}^{\mathrm{pl}} h_{m}^{\mathrm{sph}}}\right)^{2} \cos k(\omega) h_{m}^{\mathrm{sph}}\right\}, \\
& a_{22, m}(\omega)=\frac{r_{m}^{\mathrm{pl}}}{r_{m+1}^{\mathrm{pl}}} \cos k(\omega) h_{m}^{\mathrm{sph}}+\frac{r_{m+1}^{\mathrm{pl}}-r_{m}^{\mathrm{pl}}}{k(\omega) r_{m+1}^{\mathrm{pl}} h_{m}^{\mathrm{sph}}} \sin k(\omega) h_{m}^{\mathrm{sph}}
\end{aligned}
$$

with the radial conical-segment spacing

$$
h_{m}^{\mathrm{sph}}=\sqrt{\left(h^{\mathrm{pl}}\right)^{2}+\left(r_{m+1}^{\mathrm{pl}}-r_{m}^{\mathrm{pl}}\right)^{2}} .
$$

The plane-wave characteristic impedances

$$
Z_{0, m}=\frac{\rho c}{A_{m}^{\mathrm{sph}}},
$$

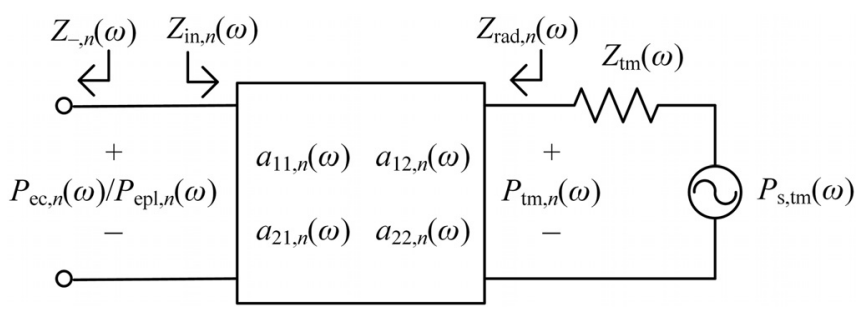

FIG. 1. The combined transmission-line and Thévenin-equivalent models used for simulating the ear canals and tympanic membrane, respectively. were calculated from the corresponding spherical-cap areas

$$
A_{m}^{\mathrm{sph}}=\left(r_{m}^{\mathrm{sph}}\right)^{2} \pi
$$

at the input of each segment with equivalent spherical-cap radii

$$
r_{m}^{\mathrm{sph}}=r_{m}^{\mathrm{pl}} \sqrt{F_{m}}
$$

Here, $F_{m}$ is the ratio of spherical-cap $A_{m}^{\mathrm{sph}}$ to planar $A_{m}^{\mathrm{pl}}$ areas (Benade, 1988),

$$
F_{m}=\frac{A_{m}^{\mathrm{sph}}}{A_{m}^{\mathrm{pl}}}=2 \frac{1-\cos \phi_{m}}{\sin ^{2} \phi_{m}}=\frac{2}{1+h^{\mathrm{pl}} / h_{m}^{\mathrm{sph}}},
$$

and $\phi_{m}$ is the taper angle of the $m$ th segment. Figure 2 shows a sketch of the concatenated conical-horn model.

We calculated the ear-canal impedances $Z_{\text {in, } n}(\omega)$ [Eq. (18)] at five equally spaced axial locations $(n=0,40,80$, 120 , and 159 with corresponding axial residual ear-canal lengths $l^{\mathrm{pl}}-n h^{\mathrm{pl}}=1.6,1.2,0.8,0.4$, and $0.1 \mathrm{~mm}$, respectively) 


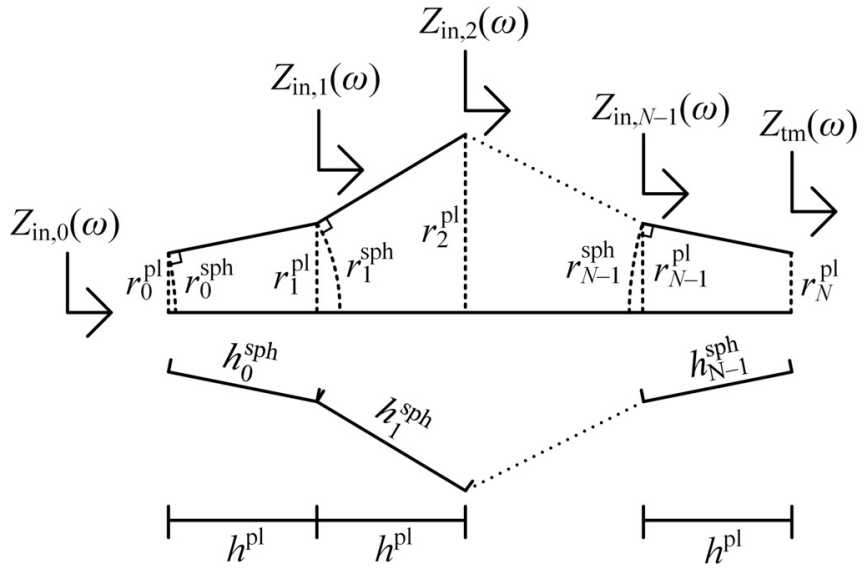

FIG. 2. A sketch illustrating the impedance and dimensional variables of the concatenated conical-horn ear-canal model, including the planar $r_{n}^{\mathrm{pl}}$ and spherical $r_{n}^{\text {sph }}$ [Eq. (27)] input radii and impedances $Z_{\text {in, }, n}(\omega)$ [Eq. (18)] of the $n$th of $N$ segments, the planar $h^{\mathrm{pl}}$ and radial $h_{n}^{\mathrm{sph}}$ [Eq. (24)] segment spacings, and the tympanic-membrane impedance $Z_{\mathrm{tm}}(\omega)$ [Eq. (37)].

and calculated the spherical-wave characteristic impedances at each location $Z_{+, n}^{\mathrm{sph}}(\omega)$ and $Z_{-, n}^{\mathrm{sph}}(\omega)$ using Eq. (10). For this purpose, the spherical-wave area gradient

$$
\left[A_{n}^{\mathrm{sph}}\right]^{\prime}=\frac{2 \pi F_{n} r_{n}^{\mathrm{pl}}\left(r_{n+1}^{\mathrm{pl}}-r_{n}^{\mathrm{pl}}\right)}{h^{\mathrm{pl}}}
$$

was calculated from the finite-difference gradient of the planar radii $r_{n+1}^{\mathrm{pl}}$ and $r_{n}^{\mathrm{pl}}$, and $F_{n}$ because it represents the rate of change in area within the same segment and not including the jump in spherical-cap area between segments. Similar to Eqs. (16) and (17), we then calculated the plane-wave reflectances

$$
\Gamma_{n}^{\mathrm{pl}}(\omega)=\frac{Z_{\text {in }, n}(\omega)-Z_{0, n}}{Z_{\text {in }, n}(\omega)+Z_{0, n}}
$$

and spherical-wave reflectances

$$
\Gamma_{n}^{\mathrm{sph}}(\omega)=\frac{Z_{\mathrm{in}, n}(\omega) / Z_{+, n}^{\mathrm{sph}}(\omega)-1}{Z_{\mathrm{in}, n}(\omega) / Z_{-, n}^{\mathrm{sph}}(\omega)+1},
$$

respectively. The corresponding time-domain reflectances $\gamma_{n}^{\mathrm{pl}}(t)$ and $\gamma_{n}^{\mathrm{sph}}(t)$ were calculated using the inverse Fourier transform $\gamma_{n}(t)=\mathcal{F}^{-1}\left[\Gamma_{n}(\omega) w(\omega) e^{j \omega \tau_{\mathrm{rt}, n}}\right]$, filtered by the one-sided low-pass half-width Blackman window $w(\omega)$ (Rasetshwane and Neely, 2011) and shifted by the radial round-trip time delay to the termination

$$
\tau_{\mathrm{rt}, n}=\frac{2}{c} \sum_{m=n}^{N-1} h_{m}^{\mathrm{sph}}
$$

to align features resulting from the reflection at the termination in the time domain at $t=0$. Time-domain impedances $z(t)$ were computed similarly, though without the time shift, $z(t)=\mathcal{F}^{-1}[Z(\omega) w(\omega)]$. We calculated the various quantities for two different cases.

(1) A simulated ear canal based on 17 points of normative planar ear-mold data reported by Egolf et al. (1993) with 1-mm spacing, but synthesized to obtain a smooth function by adding a Blackman window $w_{N, n}$ of length $N$ to a linear function and assuming axisymmetry to generate the planar radius function

$r_{n}^{\mathrm{pl}}=r_{0}^{\mathrm{pl}}+n \frac{r_{N}^{\mathrm{pl}}-r_{0}^{\mathrm{pl}}}{N}+h_{\mathrm{w}} w_{N, n}$,

where $r_{0}^{\mathrm{pl}}=4 \mathrm{~mm}, r_{N}^{\mathrm{pl}}=3.35 \mathrm{~mm}$, and $h_{\mathrm{w}}=0.9 \mathrm{~mm}$ is the window height.

(2) A simple conical horn (i.e., the same linear function without the Blackman window) to generate the radius function

$$
r_{n}^{\mathrm{pl}}=r_{0}^{\mathrm{pl}}+n \frac{r_{N}^{\mathrm{pl}}-r_{0}^{\mathrm{pl}}}{N}
$$

The axial length $l^{\mathrm{pl}}=1.6 \mathrm{~cm}$ was chosen since the data reported by Egolf et al. (1993) beyond this point from the ear-canal apex are largely uniform. We calculated the horn function $U_{n}$ [Eq. (7)] using the discrete spherical-cap radius function $r_{n}^{\text {sph }}$ by taking the finite-difference gradient twice,

$$
U_{n}=\frac{r_{n+1}^{\mathrm{sph}}-2 r_{n}^{\mathrm{sph}}+r_{n-1}^{\mathrm{sph}}}{h_{n-1}^{\mathrm{sph}} h_{n}^{\mathrm{sph}} r_{n}^{\mathrm{sph}}}
$$

Finally, for the simulated ear-canal geometry [Eq. (33)], we estimated the tympanic-membrane impedances $\hat{Z}_{\mathrm{tm}, n}(\omega)$ at each location assuming a uniform transmission-line model,

$$
\hat{Z}_{\mathrm{tm}, n}(\omega)=Z_{0, n} \frac{j Z_{0, n} \tan k(\omega)\left(l^{\mathrm{pl}}-n h^{\mathrm{pl}}\right)-Z_{\mathrm{in}, n}(\omega)}{j Z_{\mathrm{in}, n}(\omega) \tan k(\omega)\left(l^{\mathrm{pl}}-n h^{\mathrm{pl}}\right)-Z_{0, n}},
$$

where the hat operator denotes an estimated quantity. This approach allowed us to assess the influence of approximating the residual ear canal as uniform when estimating the tympanic-membrane impedance $\hat{Z}_{\mathrm{tm}, n}(\omega)$ at a distance from the tympanic membrane.

\section{B. Tympanic-membrane impedance}

To represent an acoustic load similar to the adult ear canal, we modeled the impedance of the tympanic membrane $Z_{\mathrm{tm}}(\omega)$ (see Fig. 1) as the parallel coupling of two RLC circuits,

$$
Z_{\mathrm{tm}}(\omega)=\left(R_{1}+j \omega L_{1}+\frac{1}{j \omega C_{1}}\right) \|\left(R_{2}+j \omega L_{2}+\frac{1}{j \omega C_{2}}\right) .
$$

The acoustic resistances $R_{i}$, inertances $L_{i}$, and compliances $C_{i}$ were adjusted so that $Z_{\mathrm{tm}}(\omega)$ approximated the plane-wave reflectance as measured in a Type 4157 uniform occluded-ear simulator (Brüel \& Kjær Sound \& Vibration A/S, Nærum, Denmark) of radius $r=3.75 \mathrm{~mm}$. The quantities of each $R L C$ circuit along with the corresponding natural frequencies $f_{\mathrm{n}, i}=1 /\left(2 \pi \sqrt{L_{i} C_{i}}\right)$ are listed in Table I.

\section{Stimulus-level estimation}

We investigated the utility of the plane-wave versus spherical-wave reflectances for calibrating stimulus levels, 
TABLE I. The resistances $R_{i}$, inertances $L_{i}$, compliances $C_{i}$, and corresponding natural frequencies $f_{\mathrm{n}, i}$ of the parallel $R L C$ circuits for modeling the tympanic-membrane impedance $Z_{\mathrm{tm}}(\omega)$ [Eq. (37)].

\begin{tabular}{lcccc}
\hline \hline$i$ & $R_{i}\left[\mathrm{~Pa} \mathrm{~s} / \mathrm{m}^{3}\right]$ & $L_{i}\left[\mathrm{~kg} / \mathrm{m}^{4}\right]$ & $C_{i}\left[\mathrm{~m}^{5} / \mathrm{N}\right]$ & $f_{n, I}[\mathrm{~Hz}]$ \\
\hline 1 & $6.8 \times 10^{7}$ & $1.56 \times 10^{4}$ & $1.45 \times 10^{-12}$ & 1058.2 \\
2 & $3.35 \times 10^{7}$ & 890 & $2.48 \times 10^{-12}$ & 3392.1 \\
\hline \hline
\end{tabular}

as often practiced in OAE measurements, using our analytical ear-canal models. From the total acoustic pressure at some location in the ear canal $p_{\mathrm{ec}, n}(\omega)=p_{+, n}(\omega)+p_{-, n}(\omega)$, Souza et al. (2014) calculated the forward and reverse pressure components

$$
\begin{aligned}
& p_{+, n}(\omega)=\frac{p_{\mathrm{ec}, n}(\omega)}{1+\Gamma_{n}(\omega)}, \\
& p_{-, n}(\omega)=\frac{p_{\mathrm{ec}, n}(\omega) \Gamma_{n}(\omega)}{1+\Gamma_{n}(\omega)},
\end{aligned}
$$

respectively. We estimated the pressure at the tympanic membrane as the integrated pressure (Lewis et al., 2009)

$$
p_{\text {int }, n}(\omega)=\left|p_{+, n}(\omega)\right|+\left|p_{-, n}(\omega)\right| .
$$

We referenced the plane-wave and spherical-wave integrated pressures with respect to the total sound pressure at the tympanic membrane

$$
p_{\mathrm{tm}, n}(\omega)=p_{\mathrm{ec}, n}(\omega) H_{+, n}(\omega),
$$

where the analytical forward pressure transfer function of the ear canal

$$
H_{+, n}(\omega)=\frac{p_{\mathrm{tm}, n}(\omega)}{p_{\mathrm{ec}, n}(\omega)}=\frac{Z_{\mathrm{tm}}(\omega)}{a_{11, n}(\omega) Z_{\mathrm{tm}}(\omega)+a_{12, n}(\omega)} .
$$

We evaluated these quantities at the five locations in the simulated ear canals. Although Souza et al. (2014) found that the forward pressure $p_{+, n}(\omega)$ provides superior performance for reproducing behavioral auditory thresholds, this quantity cannot be physically measured at the tympanic membrane. For the purpose of evaluating errors in stimulus-level calibration, the integrated pressure has previously been used as an estimate for the sound pressure at the tympanic membrane (Siegel et al., 2018), although it is only analytically correct for a rigid termination [because $p_{+, N}(\omega)$ and $p_{-, N}(\omega)$ are in phase when $Z_{\mathrm{tm}}(\omega) \rightarrow \infty$ ] in a uniform waveguide. For our tympanic-membrane impedance, we found that the error due to a non-rigid termination resulted in errors in the integrated pressure representing the tympanic-membrane pressure of $0.1 \mathrm{~dB}$ in a uniform ear canal. Thus, larger variations represent those of the non-uniform ear canal and the utilized characteristic impedances.

\section{Emitted pressure}

Finally, we investigated the utility of the spherical-wave characteristic impedances for computing the emitted pressure (Charaziak and Shera, 2017) from the ear. Ideally, the emitted pressure represents the sound pressure emitted from the tympanic membrane (e.g., an OAE) into an anechoic uniform ear canal. Because non-uniformities in the ear-canal geometry introduce a spatial dependence to the plane-wave emitted pressure, its calculation at different distances from the tympanic membrane may potentially be improved by using spherical-wave pressure components and terminating the ear canal using an anechoic tangent conical waveguide, i.e., the anechoic conical horn corresponding to the local cross-sectional area and area gradient. We calculated the emitted pressure $p_{\mathrm{epl}, n}(\omega)$ at the five locations in the ear canal and modeled the middle and inner ear as a Thévenin-equivalent pressure source $p_{\mathrm{s}, \mathrm{tm}}(\omega)$ (see Fig. 1). When a lossless transmission line is operated in reverse, $a_{11, n}(\omega) \leftrightarrow a_{22, n}(\omega)$. We calculated the reverse radiation impedance $Z_{\mathrm{rad}, n}(\omega)$ as seen from the tympanic membrane, terminated by the plane-wave or reverse-spherical-wave characteristic impedance $Z_{-, n}(\omega)$ at the given locations,

$$
Z_{\mathrm{rad}, n}(\omega)=\frac{a_{22, n}(\omega) Z_{-, n}(\omega)+a_{12, n}(\omega)}{a_{21, n}(\omega) Z_{-, n}(\omega)+a_{11, n}(\omega)} .
$$

We then calculated the pressure at the tympanic membrane $p_{\mathrm{tm}, n}(\omega)$ as the voltage division of $p_{\mathrm{s}, \mathrm{tm}}(\omega)$ between $Z_{\mathrm{tm}}(\omega)$ and $Z_{\mathrm{rad}, n}(\omega)$,

$$
p_{\mathrm{tm}, n}(\omega)=p_{\mathrm{s}, \mathrm{tm}}(\omega) \frac{Z_{\mathrm{rad}, n}(\omega)}{Z_{\mathrm{tm}}(\omega)+Z_{\mathrm{rad}, n}(\omega)},
$$

and transformed it to the emitted pressure $p_{\mathrm{epl}, n}(\omega)$ at a given location using the reverse pressure transfer function $H_{-, n}(\omega)$ between the tympanic membrane and the given locations,

$$
p_{\mathrm{epl}, n}(\omega)=p_{\mathrm{tm}, n}(\omega) H_{-, n}(\omega),
$$

where

$$
H_{-, n}(\omega)=\frac{p_{\mathrm{epl}, n}(\omega)}{p_{\mathrm{tm}, n}(\omega)}=\frac{Z_{-, n}(\omega)}{a_{22, n}(\omega) Z_{-, n}(\omega)+a_{12, n}(\omega)} .
$$

We referenced $p_{\text {epl, },}(\omega)$ against the emitted pressure $p_{\text {epl,ref }}(\omega)$ looking into a uniform waveguide of radius $r=3.75 \mathrm{~mm}$ [identical to the occluded-ear simulator used for deriving the tympanic-membrane impedance $Z_{\mathrm{tm}}(\omega)$ ]. Ideally, the emitted pressure would represent that into the plane-wave characteristic impedance of a uniform ear canal, but this approach allows us to simulate the variation that occurs due to the presence of a non-uniform ear canal and by employing the plane-wave and spherical-wave characteristic impedances for quantifying the emitted pressure.

\section{NUMERICAL RESULTS}

\section{A. Ear-canal reflectance}

Figures 3(a) and 3(b) show the planar radius functions $r_{n}^{\mathrm{pl}}$ [Eqs. (33) and (34)] and corresponding horn functions $U_{n}$ [Eq. (35)], respectively, for both the simulated ear canal and conical waveguide. In addition, Fig. 3(a) shows the segment 
(a)

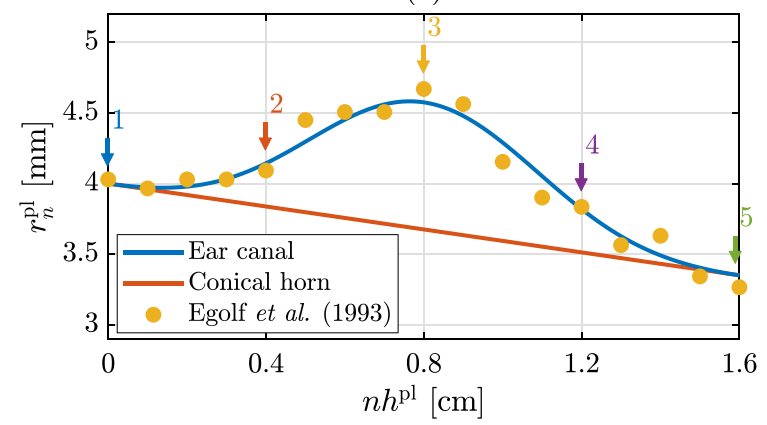

(c) Ear canal

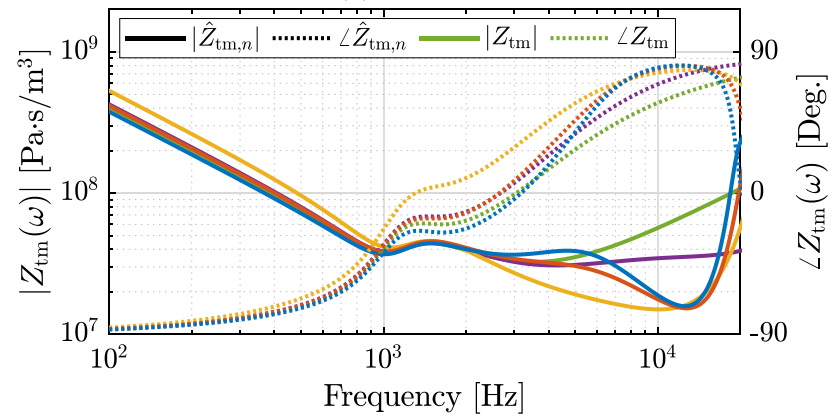

(e) Ear canal

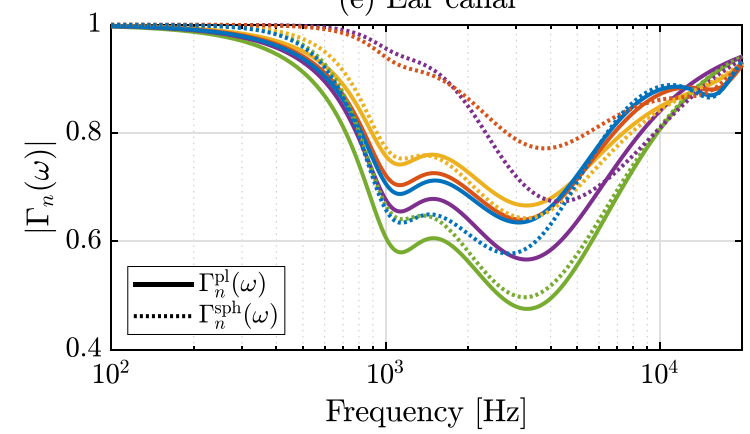

(g) Conical horn

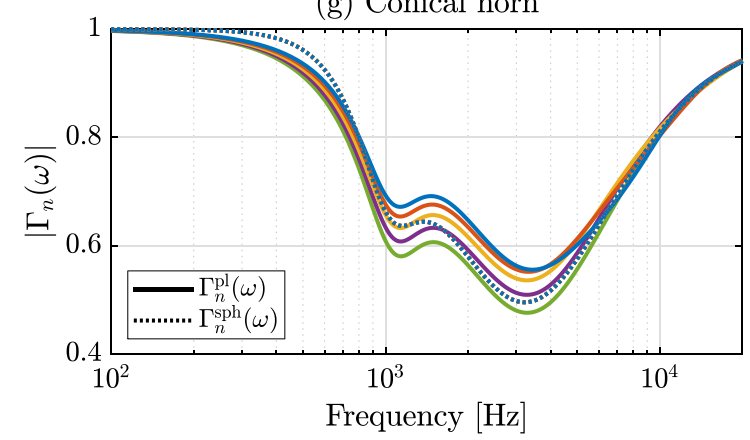

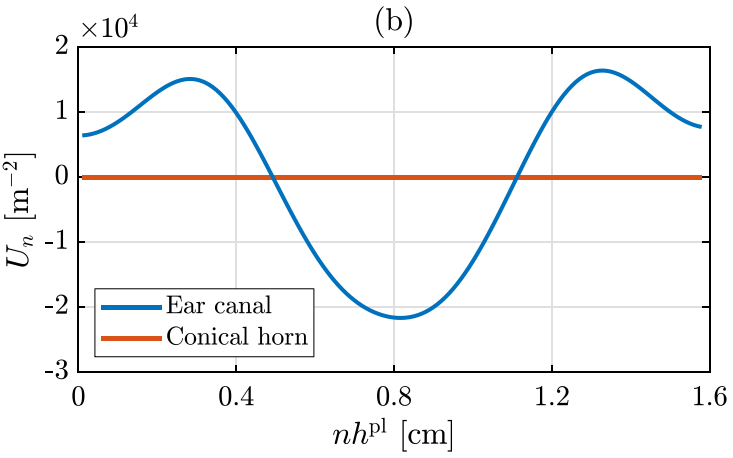

(d) Ear canal

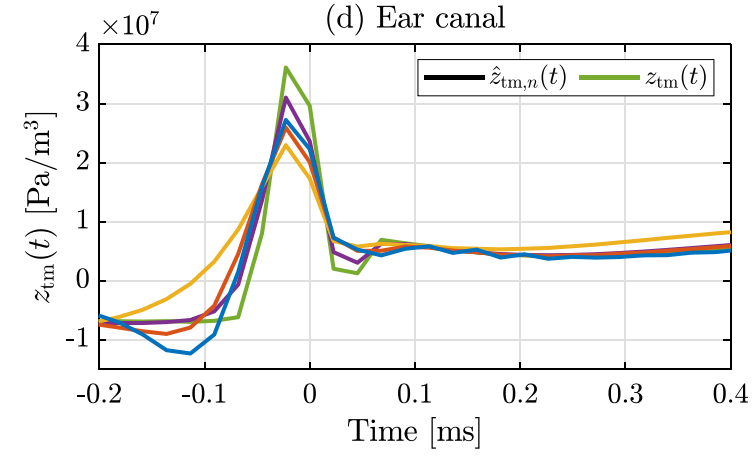

(f) Ear canal

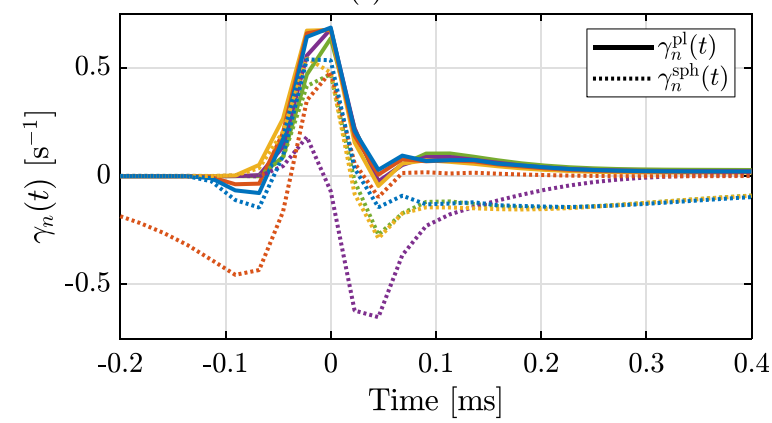

(h) Conical horn

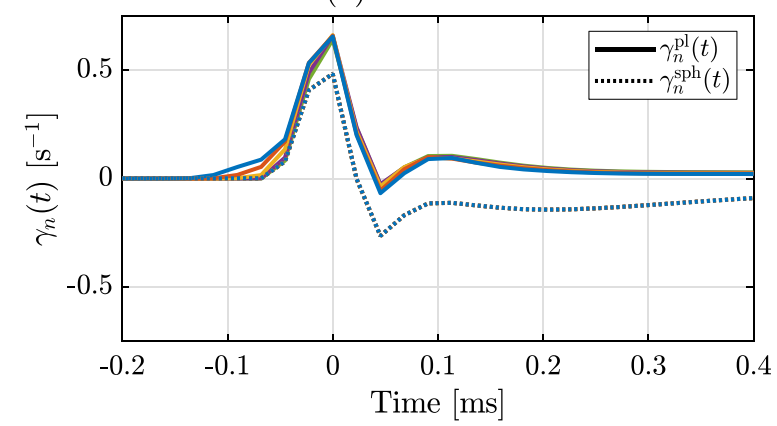

FIG. 3. (Color online) The (a) planar radius functions $r_{n}^{\mathrm{pl}}$ [Eqs. (33) and (34)] and (b) horn functions $U_{n}$ [Eq. (35)] of the simulated ear canal, based on ear-mold data from Egolf et al. (1993), and conical waveguide, (c) magnitude and phase of the estimated $\hat{Z}_{\mathrm{tm}, n}(\omega)$ [Eq. (36)] and actual $Z_{\mathrm{tm}}(\omega)$ [Eq. (37)] tympanicmembrane impedances, and (d) corresponding time-domain impedances $\hat{z}_{\mathrm{tm}, n}(t)$ and $z_{\mathrm{tm}}(t)$ in the ear canal. The (e),(g) plane-wave $\left|\Gamma_{n}^{\mathrm{pl}}(\omega)\right|[\mathrm{Eq}$. (30)] and spherical-wave $\left|\Gamma_{n}^{\mathrm{sph}}(\omega)\right|$ [Eq. (31)] reflectance magnitudes, and (f),(h) corresponding time-domain reflectances $\gamma_{n}^{\mathrm{pl}}(t)$ and $\gamma_{n}^{\mathrm{sph}}(t)$ of the (e),(f) ear canal and (g),(h) conical horn. Each color in (c)-(h) corresponds to locations 1-5 marked in (a), although we show $Z_{\mathrm{tm}}(\omega)$ and $z_{\mathrm{tm}}(t)$ for location 5 in (c) and (d), respectively.

of discrete data points reported by Egolf et al. (1993) on which the ear-canal model was based. For the locations 1-5 marked in Fig. 3(a), the plane-wave $\Gamma_{n}^{\mathrm{pl}}(\omega)$ [Eq. (30)] and spherical-wave $\Gamma_{n}^{\mathrm{sph}}(\omega)$ [Eq. (31)] reflectances, and corresponding time-domain reflectances $\gamma_{n}^{\mathrm{pl}}(t)$ and $\gamma_{n}^{\mathrm{sph}}(t)$ were calculated, each color in Figs. 3(c)-3(h) representing the location color and number marked in Fig. 3(a). For the simulated ear canal, Figs. 3(c) and 3(d) show the magnitude and phase of the estimated $\hat{Z}_{\mathrm{tm}, n}(\omega)$ [Eq. (36), i.e., locations 1-4] and actual $Z_{\mathrm{tm}}(\omega)$ [Eq. (37), i.e., location 5] tympanicmembrane impedances, and the corresponding time-domain impedances $\hat{z}_{\mathrm{tm}, n}(t)$ and $z_{\mathrm{tm}}(t)$, respectively. These plots indicate the expected error when estimating the tympanicmembrane impedance using a uniform transmission-line model and show that the model may be inadequate at higher frequencies. The step at $z_{\mathrm{tm}}(t=0)$ is a result of the low-frequency compliant behavior in $Z_{\mathrm{tm}}(\omega) . U_{n}$ varies substantially along the canal because of the complex geometry of the simulated ear 
canal and is sometimes negative. At frequencies below the critical frequency $\omega_{c}$ [Eqs. (14) and (15)], pressure waves become evanescent and non-propagating. As expected, $U_{n}=0$ throughout the conical horn.

For the simulated ear canal, Fig. 3(e) shows $\left|\Gamma_{n}^{\mathrm{pl}}(\omega)\right|$ and $\left|\Gamma_{n}^{\mathrm{sph}}(\omega)\right|$ and Fig. 3(f) shows $\gamma_{n}^{\mathrm{pl}}(t)$ and $\gamma_{n}^{\mathrm{sph}}(t)$ for the five locations. The plots show that both $\left|\Gamma_{n}^{\mathrm{sph}}(\omega)\right|$ and $\gamma_{n}^{\mathrm{sph}}(t)$ depend sensitively on $n$ due to the influence of the areafunction gradient on the spherical-wave characteristic impedances $Z_{ \pm, n}^{\text {sph }}(\omega)$ [Eq. (10)]. The plane-wave reflectances $\left|\Gamma_{n}^{\mathrm{pl}}(\omega)\right|$ and $\gamma_{n}^{\mathrm{pl}}(t)$ are less sensitive to location, and $\gamma_{n}^{\mathrm{pl}}(t)$ more accurately represent the overall features in $z_{\mathrm{tm}}(t)$. The figures reveal how $\gamma_{n}^{\text {sph }}(t)$ are affected by causal or noncausal exponential components due to the time-domain spherical-wave characteristic impedances $z_{ \pm, n}^{\mathrm{sph}}(t)$ [Eq. (13)], represented as the apparent exponential decays in $\gamma_{n}^{\mathrm{sph}}(t)$. These exponential components, which substantially limit time-domain analysis of the spherical-wave reflectance, arise due to the large changes in wavefront shape along the ear canal relative to the wavefront shape in an equivalent anechoic conical horn corresponding to the local wavefrontarea gradient. Aside from these exponential components, the time-domain spherical-wave reflectances $\gamma_{n}^{\text {sph }}(t)$ largely resemble the time-domain plane-wave reflectances $\gamma_{n}^{\mathrm{pl}}(t)$. In the frequency domain, the reflection from the termination is contaminated by the local area gradient in $\left|\Gamma_{n}^{\mathrm{sph}}(\omega)\right|$ and is therefore more consistently represented in $\left|\Gamma_{n}^{\mathrm{pl}}(\omega)\right|$. Note that the results at location 5 may not represent the desired planewave reflectance of the tympanic membrane. Because of the oblique angle of the tympanic membrane relative to the ear

(a) Ear canal

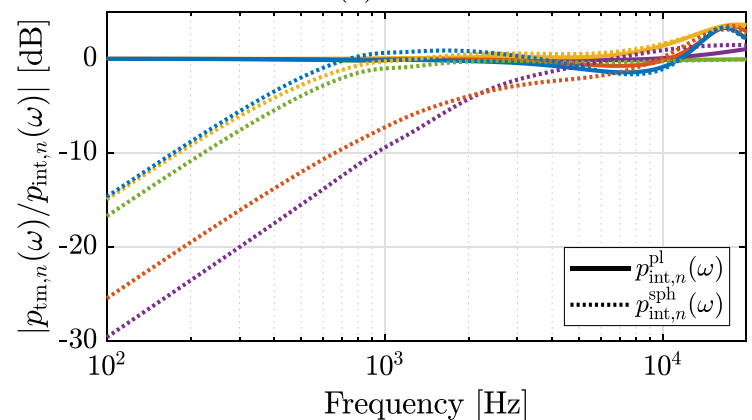

(c) Ear canal

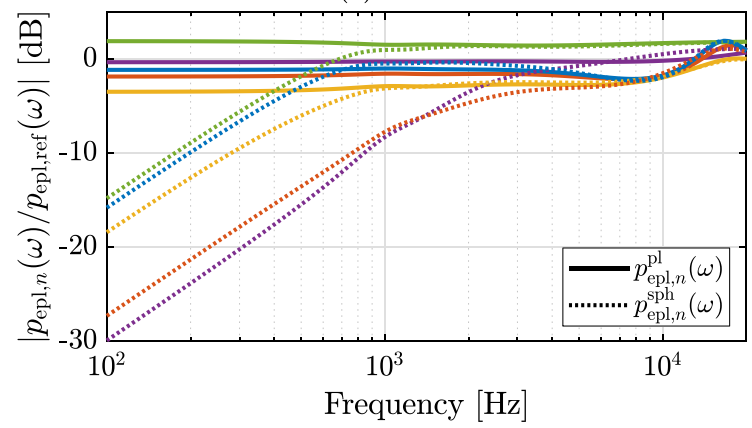

canal, the ear-canal cross-sectional area closer to the tympanic membrane decreases, although the surface of the tympanic membrane is larger [see Fig. 8 of Egolf et al. (1993)].

For the conical horn, Fig. 3(g) shows $\left|\Gamma_{n}^{\mathrm{pl}}(\omega)\right|$ and $\left|\Gamma_{n}^{\mathrm{sph}}(\omega)\right|$, and Fig. 3(h) shows $\gamma_{n}^{\mathrm{pl}}(t)$ and $\gamma_{n}^{\mathrm{sph}}(t)$ for the five locations. From these results, the virtues of the sphericalwave characteristic impedances can be appreciated since $\left|\Gamma_{n}^{\mathrm{sph}}(\omega)\right|$ and $\gamma_{n}^{\mathrm{sph}}(t)$ are now insensitive to location, while $\left|\Gamma_{n}^{\mathrm{pl}}(\omega)\right|$ decreases systematically with distance and $\gamma_{n}^{\mathrm{pl}}(t)$ changes only marginally. Although the termination contributes similarly to $\gamma_{n}^{\mathrm{pl}}(t)$ and $\gamma_{n}^{\mathrm{sph}}(t),\left|\Gamma_{n}^{\mathrm{pl}}(\omega)\right|$ and $\left|\Gamma_{n}^{\mathrm{sph}}(\omega)\right|$ differ substantially and $\left|\Gamma_{n}^{\mathrm{sph}}(\omega)\right|$ do generally not characterize the plane-wave reflectance of the termination (location 5).

\section{B. Stimulus- and emitted-pressure-level estimation}

For the five locations in the simulated ear canal and conical horn, Figs. 4(a) and 4(b), respectively, show the magnitude of pressures at the tympanic membrane $p_{\mathrm{tm}, n}(\omega)$ [Eq. (41)], referenced to the integrated pressures $p_{\text {int }, n}^{\mathrm{pl}}(\omega)$ and $p_{\text {int }, n}^{\text {sph }}(\omega)$ [Eq. (40)] computed using the plane-wave $\Gamma_{n}^{\mathrm{pl}}(\omega)$ [Eq. (30)] and spherical-wave $\Gamma_{n}^{\mathrm{sph}}(\omega)$ [Eq. (31)] reflectances, respectively. The ratio $\left|p_{\mathrm{tm}, n}(\omega) / p_{\text {int }, n}(\omega)\right|$ can then directly be interpreted as a stimulus error [we find a qualitatively similar pattern of errors when using the ratio $\left.\left|p_{\mathrm{tm}, n}(\omega) / p_{+, n}(\omega)\right|\right]$. While $p_{\mathrm{int}, n}^{\mathrm{pl}}(\omega)$ approach $p_{\mathrm{tm}, n}(\omega)$ at low frequencies as expected, $p_{\text {int }, n}^{\mathrm{sph}}(\omega)$ deviate substantially and do not consistently approximate the sound pressure at the tympanic membrane throughout the canal. This is because the spherical-wave characteristic impedances $Z_{ \pm, n}^{\mathrm{sph}}(\omega)$ [Eq. (10)] have small magnitudes (due to the parallel inertance)

(b) Conical horn

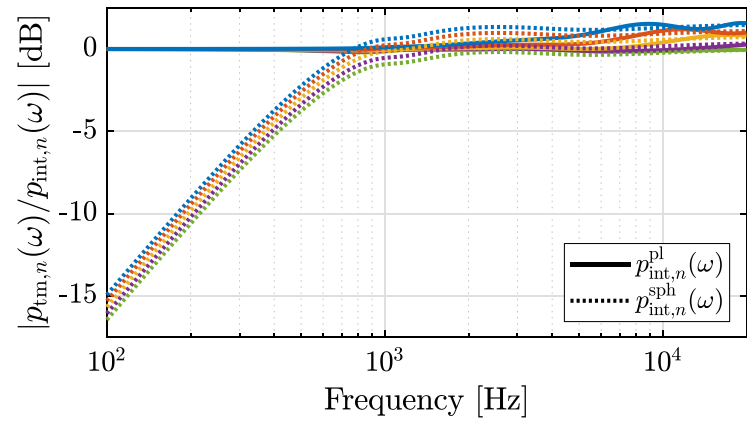

(d) Conical horn

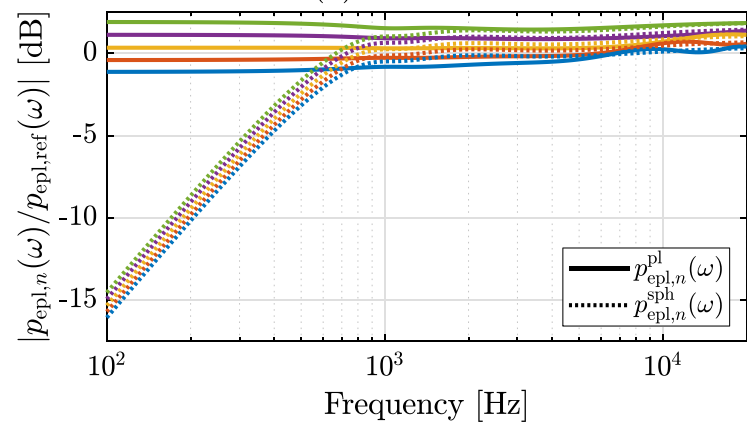

FIG. 4. (Color online) (a),(b) The pressures at the tympanic membrane $p_{\mathrm{tm}, n}(\omega)$ [Eq. (41)] referenced against the integrated pressures $p_{\mathrm{int}, n}^{\mathrm{pl}}(\omega)$ and $p_{\mathrm{int}, n}^{\mathrm{sph}}(\omega)$ [Eq. (40)] computed using the plane-wave $\Gamma_{n}^{\mathrm{pl}}(\omega)$ [Eq. (30)] and spherical-wave $\Gamma_{n}^{\text {sph }}(\omega)$ [Eq. (31)] reflectances, respectively. (c),(d) The emitted pressures $p_{\mathrm{epl}, n}^{\mathrm{pl}}(\omega)$ and $p_{\mathrm{epl}, n}^{\mathrm{sph}}(\omega)$ [Eq. (44)] computed using the reverse plane-wave $Z_{-, n}^{\mathrm{pl}}(\omega)=Z_{0, n}$ [Eq. (25)] and spherical-wave $Z_{-, n}^{\mathrm{sph}}(\omega)$ [Eq. (10)] characteristic impedances, respectively, referenced against the emitted pressure $p_{\text {epl,ref }}(\omega)$ into a uniform waveguide of radius $r=3.75 \mathrm{~mm}$. Results are shown for the five locations marked in Fig. 3(a) for the simulated (a),(c) ear canal and (b),(d) conical horn. 
and opposite phases at low frequencies, which cause the forward $p_{+, n}(\omega)$ and reverse $p_{-, n}(\omega)$ pressures to have large magnitudes and opposite phases. In addition, the forward and reverse pressures of a spherical wave are not in phase at the termination, which is an assumption made when performing in-situ calibrations using the integrated pressure. The consequence of this in OAE measurements would be a substantial under stimulation. In the conical horn, $p_{\mathrm{int}, n}^{\mathrm{sph}}(\omega)$ are vertically offset from another at the various locations, indicating that they differ by a constant factor. This discrepancy arises because the integrated pressure does not take into account the inverse magnitude dependency of the pressure propagating in the horn. At high frequencies, $p_{\mathrm{int}, n}^{\mathrm{pl}}(\omega)$ and $p_{\text {int }, n}^{\text {sph }}(\omega)$ perform similarly because $Z_{ \pm, n}^{\text {sph }}(\omega)$ approach $Z_{0, n}$.

For the five locations in the simulated ear canal and conical horn, Figs. 4(c) and 4(d), respectively, show the emitted pressures $p_{\mathrm{epl}, n}^{\mathrm{pl}}(\omega)$ and $p_{\mathrm{epl}, n}^{\mathrm{sph}}(\omega)$ [Eq. (44)] computed using the reverse plane-wave $Z_{-, n}^{\mathrm{pl}}(\omega)=Z_{0, n}$ and spherical-wave $Z_{-, n}^{\mathrm{sph}}(\omega)$ characteristic impedances, respectively. The results are normalized by the emitted pressure $p_{\text {epl,ref }}(\omega)$ obtained in a uniform waveguide of radius $r=3.75 \mathrm{~mm}$. Recall here that the virtue of the emitted pressure is an independence of location in the ear canal rather than constancy of absolute level. The general deviation of $p_{\mathrm{epl}, n}^{\mathrm{sph}}(\omega)$ from $p_{\mathrm{epl}, \mathrm{ref}}(\omega)$ is, similar to the integrated pressure, a result of the parallel inertance in $Z_{-, n}^{\mathrm{sph}}(\omega)$. Despite this, $p_{\mathrm{epl}, n}^{\mathrm{pl}}(\omega)$ does vary less than $p_{\mathrm{epl}, n}^{\mathrm{sph}}(\omega)$ at low frequencies in the simulated ear canal. However, in the conical waveguide, there is no variation in $p_{\mathrm{epl}, n}^{\mathrm{sph}}(\omega)$ between the various locations except for that due to the inverse magnitude dependency.

\section{DISCUSSION}

Although the spherical-wave characteristic impedances can be useful for analyzing conical horns, they possess properties that limit their application in the practical case of earcanal reflectance. The tangent conical horn at the location of a large taper in the simulated ear canal provides a poor representation of the remaining ear-canal geometry and the instability of the spherical-wave characteristic impedances limits their utility for analyzing results in the time domain using the inverse Fourier transform. These properties also have negative implications for calibrating stimulus levels and estimating the emitted pressure using existing methods, which are largely based on the assumption of plane waves. Existing plane-wave ear-canal-reflectance measurement methods require estimating the plane-wave characteristic impedance of the ear canal in situ. Practical application of the sphericalwave reflectance in ear canals is thus further complicated by the need to estimate the inertance representing the inverse wavefront area gradient.

The Blackman window and time delay applied to the time-domain reflectances introduce some degree of noncausality into the synthesized time-domain signals. However, these and the non-causality due to the spherical-wave characteristic impedances do not affect the validity of the frequency-domain results because they are solely artifacts resulting from the inverse Fourier transform and represent what Nørgaard et al. (2018) refer to as a signal non-causality, rather than a physical non-causality. Thus, the reported timedomain quantities satisfying causality cannot be interpreted as a condition for the validity of the remaining frequencydomain quantities. Causal time-domain reflectances might be obtained using a time-domain model as the starting point.

The horn functions indicate whether propagating waves exist at a given location and frequency. For frequencies below and around the critical frequency, the solutions to the reduced horn equation transition from propagating to evanescent waves. In these regions, the concept of reverse- and forward-propagating pressure components lose their intuitive meaning, and the characteristic impedances used for separating these components may ultimately come down to choice. In the considered analytical examples, the spherical-wave characteristic impedances arguably yield the correct earcanal reflectance in its formal definition (the ratio of reverseto forward-propagating pressures) because the simulated ear canals were constructed from concatenated conical horn segments. However, since the sound wavelength is much longer than each horn segment, the results are practically identical to those of a continuous area function. Note that the horn function depends on the second derivative of the area function whereas the inertance in the spherical-wave characteristic impedances depends on its first derivative. For locations corresponding to an area-gradient extremum, the sphericalwave reflectance represents the true ratio of independently propagating pressure components despite providing the poorest representation of the reflection from the tympanic membrane.

Contributions to the reflectance due to area variations along a non-uniform lossless waveguide are caused by deviations in the wavefront area from the geometry implicitly assumed by the characteristic impedances (e.g., of the tangent anechoic conical horn corresponding to the local wavefrontarea gradient). Employing the spherical-wave characteristic impedances at the location of a large area gradient results in exponentially decaying components along the simulated ear canal in the time-domain reflectance (because the overall geometry of the canal is poorly represented by the local gradient). Were the ear canal approximately conical in its overall shape, the spherical-wave reflectance might constitute an improvement. However, the reflection of incident plane and spherical waves on the same tympanic-membrane impedance still differs substantially and the latter contributes with exponential reflection components. This greatly contaminates the representation of reflections from the tympanic membrane and, consequently, the spherical-wave reflectance provides no improvement in characterizing the sound reflected from the middle ear. Due to the time-domain representation of the plane-wave characteristic impedance [Eq. (9)], the various features of the time-domain tympanic-membrane impedance are directly represented in the time-domain plane-wave reflectance. Our results show that if one wishes to characterize the plane-wave reflectance of the tympanic membrane at a distance, one is better off utilizing the plane-wave characteristic impedance for calculating the reflectance. An 
unavoidable consequence, however, is that non-uniformities between the measurement location and the tympanic membrane contribute to the plane-wave reflectance. Avoiding these contributions requires compensating for the nonuniform ear canal [e.g., using a transmission-line model to obtain the impedance at or closer to the tympanic membrane (Lewis and Neely, 2015)], for which accurate knowledge of ear-canal geometry is needed. Our results show that the assumption of a uniform ear canal is often inaccurate for this application.

In recent years, reflectance-based methods for calibrating acoustic stimuli have been used extensively in research for calibrating stimulus levels. The forward pressure is the most frequently used quantity, mostly as a result of the study by Souza et al. (2014), which showed that this quantity performs well for minimizing the variation in behavioral auditory thresholds. Of course, with knowledge of ear-canal geometry, the pressure at the measurement location could be transformed into sound pressure at the tympanic membrane using the ear-canal transmission-line matrix. Even in this case, however, the sound pressure at the tympanic membrane may not accurately predict the stimulation of the cochlea.

\section{CONCLUSION}

In this paper, we have investigated the application of the spherical-wave characteristic impedances for calculating the ear-canal reflectance, characterizing the conductive status of the middle ear, calibrating stimulus levels, and estimating the emitted pressure from the ear. Relevant properties of the spherical-wave characteristic impedances have been reviewed and, despite certain virtues, they were found to limit both the frequency- and time-domain analysis of the spherical-wave ear-canal reflectance. The application of the spherical-wave characteristic impedances, identical to those of the WKB approximation, was investigated in analytical loads similar to the human ear canal. Although the sphericalwave characteristic impedances provide the most accurate decomposition of the total acoustic pressure into its forwardand reverse-propagating components, time-domain exponential reflection components obscure the reflections from the tympanic membrane. To best characterize the plane-wave reflectance of the tympanic membrane in the ear canal, one should evidently utilize the plane-wave characteristic impedance, with the caveat that the plane-wave reflectance will include contributions from the non-uniform ear canal.

\section{ACKNOWLEDGMENTS}

The authors thank the anonymous reviewers for valuable comments on this and a previous version of this manuscript. This research was funded by the Innovation Fund Denmark, Grant No. 5189-00100B (K.R.N.), the National Institutes of Health, Grant Nos. K99 DC016906 (K.K.C.) and R01 DC003687 (C.A.S.), and Interacoustics A/S.

${ }^{1}$ In the field of physiological acoustics, the reflection coefficient is usually denoted as $R$. In other fields, $\Gamma$ is often standard notation and we chose $\Gamma$ here to avoid confusion with other variables.
${ }^{2}$ Note that some authors define the forward and reverse volume flows as $u_{ \pm}(\omega, x)$ [opposed to our choice of $\pm u_{ \pm}(\omega, x)$ ], whereby the total acoustic volume flow is $u(\omega, x)=u_{+}(\omega, x)-u_{-}(\omega, x)$ and leaving Eq. (4) unchanged.

${ }^{3}$ Despite of these solutions being exact, they do not necessarily provide an accurate approximation of the behavior of a physical horn.

${ }^{4}$ Benade (1988) reported the spherical-wave characteristic impedances $Z_{ \pm}^{\mathrm{sph}}(\omega, x)$ as a function of the apical distances $x_{0}$ of the conical horn. The spherical-cap area-function derivative at the apical distance is

$$
\left[A^{\mathrm{sph}}\left(x_{0}\right)\right]^{\prime}=\frac{2 A^{\mathrm{sph}}\left(x_{0}\right)}{x_{0}} .
$$

${ }^{5}$ Agulló et al. (1988) inadvertently reported obtaining the time-domain spherical-wave characteristic impedances $z_{ \pm}^{\text {sph }}(t, x)$ in Eq. (12) using the Fourier transform despite showing the result of the unilateral Laplace transform [Eq. (13)], i.e.,

$$
z_{ \pm}^{\mathrm{sph}}(t, x)=\mathcal{F}^{-1}\left[Z_{ \pm}^{\mathrm{sph}}(\omega, x)\right]=Z_{0}(x)\left(\delta(t) \mp \frac{e^{\mp t / \tau(x)}}{\tau(x)} \theta(t)\right) .
$$

${ }^{6}$ Some authors refer to the plane-wave characteristic impedance $Z_{0}$ as simply the characteristic impedance in a more general sense, and the spherical-wave characteristic impedances $Z_{ \pm}^{\text {sph }}(\omega, x)$ (or the characteristic impedances of other types of horns) as the radiation impedance of the anechoic horn.

Agulló, J., Barjau, A., and Keefe, D. H. (1999). "Acoustic propagation in flaring, axisymmetric horns: I. A new family of unidimensional solutions," Acta Acust. United Acust. 85, 278-284.

Agulló, J., Barjau, A., and Martínez, J. (1988). "Alternatives to the impulse response $h(t)$ to describe the acoustical behavior of conical ducts," J. Acoust. Soc. Am. 84, 1606-1612.

Agulló, J., Barjau, A., and Martínez, J. (1990). "A comment on 'Conical bores. Part I: Reflection functions associated with discontinuities' [J. Acoust. Soc. Am. 84, 1613-1619 (1988)] and 'Alternatives to the impulse response $h(t)$ to describe the acoustical behavior of conical ducts' [J. Acoust. Soc. Am. 84, 1606-1612 (1988)]," J. Acoust. Soc. Am. 87, 904.

Benade, A. H. (1988). "Equivalent circuits for conical waveguides," J. Acoust. Soc. Am. 83, 1764-1769.

Benade, A. H., and Jansson, E. V. (1974). "On plane and spherical waves in horns with nonuniform flare I. Theory of radiation, resonance frequencies and mode conversion," Acustica 31, 79-98.

Caussé, R., Kergomard, J., and Lurton, X. (1984). "Input impedance of brass musical instruments-Comparison between experiment and numerical models," J. Acoust. Soc. Am. 75, 241-254.

Charaziak, K. K., and Shera, C. A. (2017). "Compensating for ear-canal acoustics when measuring otoacoustic emissions," J. Acoust. Soc. Am. 141, 515-531.

Egolf, D. P., Nelson, D. K., Howell, H. C., and Larson, V. D. (1993). "Quantifying ear-canal geometry with multiple computer-assisted tomographic scans," J. Acoust. Soc. Am. 93, 2809-2819.

Ellison, J. C., Gorga, M., Cohn, E., Fitzpatrick, D., Sanford, C. A., and Keefe, D. H. (2012). "Wideband acoustic transfer functions predict middle-ear effusion," Laryngoscope 122, 887-894.

Farmer-Fedor, B. L., and Rabbitt, R. D. (2002). "Acoustic intensity, impedance and reflection coefficient in the human ear canal," J. Acoust. Soc. Am. 112, 600-620.

Feeney, M. P., and Keefe, D. H. (2001). "Estimating the acoustic reflex threshold from wideband measures of reflectance, admittance, and power," Ear Hear. 22, 316-332.

Gilbert, J., Kergomard, J., and Polack, J. D. (1990). "On the reflection functions associated with discontinuities in conical bores," J. Acoust. Soc. Am. 87, 1773-1780.

Keefe, D. H., and Barjau, A. (1999). "Acoustic propagation in flaring, axisymmetric horns: II. Numerical results, WKB theory, and viscothermal effects," Acta Acust. United Acust. 85, 285-293.

Keefe, D. H., Folsom, R. C., Gorga, M. P., Vohr, B. R., Bulen, J. C., and Norton, S. J. (2000). "Identification of neonatal hearing impairment: Earcanal measurements of acoustic admittance and reflectance in neonates," Ear Hear. 21, 443-461. 
Keefe, D. H., Ling, R., and Bulen, J. C. (1992). "Method to measure acoustic impedance and reflection coefficient," J. Acoust. Soc. Am. 91, 470-485.

Keefe, D. H., Sanford, C. A., Ellison, J. C., Fitzpatrick, D. F., and Gorga, M. P. (2012). "Wideband aural acoustic absorbance predicts conductive hearing loss in children," Int. J. Audiol. 51, 880-891.

Leach, W. M., Jr. (1996). "A two-port analogous circuit and SPICE model for Salmon's family of acoustic horns," J. Acoust. Soc. Am. 99, 1459-1464.

Lewis, J. D., McCreery, R. W., Neely, S. T., and Stelmachowicz, P. G. (2009). "Comparison of in-situ calibration methods for quantifying input to the middle ear," J. Acoust. Soc. Am. 126, 3114-3124.

Lewis, J. D., and Neely, S. T. (2015). "Non-invasive estimation of middleear input impedance and efficiency," J. Acoust. Soc. Am. 138, 977-993.

Martínez, J., and Agulló, J. (1988). "Conical bores. Part I: Reflection functions associated with discontinuities," J. Acoust. Soc. Am. 84, 1613-1619.

Martínez, J., Agulló, J., and Cardona, S. (1988). "Conical bores. Part II: Multiconvolution," J. Acoust. Soc. Am. 84, 1620-1627.

McCreery, R. W., Pittman, A., Lewis, J., Neely, S. T., and Stelmachowicz, P. G. (2009). "Use of forward pressure level to minimize the influence of acoustic standing waves during probe-microphone hearing-aid verification," J. Acoust. Soc. Am. 126, 15-24.

Merchant, G. R., Röösli, C., Niesten, M. E. F., Hamade, M. A., Lee, D. J., McKinnon, M. L., Ulku, C. H., Rosowski, J. J., Merchant, S. N., and Nakajima, H. H. (2014). "Power reflectance as a screening tool for the diagnosis of superior semicircular canal dehiscence," Otol. Otolaryngol. 36, 172-177.

Nørgaard, K. R., Fernandez-Grande, E., and Laugesen, S. (2017). "Compensating for evanescent modes and estimating characteristic impedance in waveguide acoustic impedance measurements," J. Acoust. Soc. Am. 142, 3497-3509.

Nørgaard, K. R., Neely, S. T., and Rasetshwane, D. M. (2018). "Quantifying undesired parallel components in Thévenin-equivalent acoustic source parameters," J. Acoust. Soc. Am. 143, 1491-1503.
Piskorski, P., Keefe, D. H., Simmons, J. L., and Gorga, M. P. (1999). "Prediction of conductive hearing loss based on acoustic ear-canal response using a multivariate clinical decision theory," J. Acoust. Soc. Am. 105, 1749-1764.

Putland, G. R. (1993). "Every one-parameter acoustic field obeys Webster's horn equation,” J. Audio Eng. Soc. 41, 435-451.

Rasetshwane, D. M., and Neely, S. T. (2011). "Inverse solution of ear-canal area function from reflectance," J. Acoust. Soc. Am. 130, 3873-3881.

Salmon, V. (1946a). "A new family of horns," J. Acoust. Soc. Am. 17, $212-218$.

Salmon, V. (1946b). "Generalized plane wave horn theory," J. Acoust. Soc. Am. 17, 199-211.

Scheperle, R. A., Goodman, S. S., and Neely, S. T. (2011). "Further assessment of forward pressure level for in situ calibration," J. Acoust. Soc. Am. 130, 3882-3892.

Scheperle, R. A., Neely, S. T., Kopun, J. G., and Gorga, M. P. (2008). "Influence of in situ, sound-level calibration on distortion-product otoacoustic emission variability," J. Acoust. Soc. Am. 124, 288-300.

Siegel, J. H., Nørgaard, K. R., and Neely, S. T. (2018). "Evanescent waves in simulated ear canals: Experimental demonstration and method for compensation," J. Acoust. Soc. Am. 144, 2135-2146.

Souza, N. N., Dhar, S., Neely, S. T., and Siegel, J. H. (2014). "Comparison of nine methods to estimate ear-canal stimulus levels," J. Acoust. Soc. Am. 136, 1768-1787.

Voss, S. E., and Allen, J. B. (1994). "Measurement of acoustic impedance and reflectance in the human ear canal," J. Acoust. Soc. Am. 95, 372-384.

Webster, A. G. (1919). "Acoustical impedance, and the theory of horns and of the phonograph," Proc. Natl. Acad. Sci. 5, 275-282.

Withnell, R. H., Jeng, P. S., Waldvogel, K., Morgenstein, K., and Allen, J. B. (2009). "An in situ calibration for hearing thresholds," J. Acoust. Soc. Am. 125, 1605-1611. 